\title{
A Rare Presentation of Systemic Lupus Erythematosus during Childhood: Chorea
}

\section{Ayşegül DOĞAN DEMiR ${ }^{1}$, Mehmet KÜÇÜKKOÇ², Nilüfer GÖKNAR ${ }^{3}$, Türkan UYGUR ŞAHIN', Ufuk ERENBERK'}

${ }^{1}$ Department of Pediatrics, Bezmialem Vakıf University, İstanbul, Turkey

${ }^{2}$ Clinic of Pediatrics, Nizip State Hospital, Public Hospitals Administration of Turkey, Gaziantep, Turkey

${ }^{3}$ Clinic of Pediatric Nephrology, Bağclar Training and Research Hospital, İstanbul, Turkey

\section{ABSTRACT}

Systemic lupus erythematosus (SLE) is a systemic, chronic, inflammatory, autoimmune disease characterized by multi-organ and -system involvement such as the kidneys and hematologic and central nervous systems. The clinical presentation of the disease is extremely variable. Systemic findings, nephritis, neuropsychiatric disease, and cytopenia are more common in children than in adults at presentation. Chorea typically manifests with jerky, involuntary, and purposeless movements, involving the face, four limbs, and neck. In addition, patients suffer from dysarthria, gait disturbance, and neuropsychiatric symptoms. Chorea can be the first finding of SLE during childhood. Herein we reported a 13-year female patient who presented with movement and speech disorders and who was diagnosed chorea-related SLE.

Keywords: Systemic lupus erythematosus, chorea, children

\section{Introduction}

Systemic lupus erythematosus (SLE) is a chronic autoimmune disease that affects almost all body systems, particularly the kidneys, skin, hematological system, and central nervous system. It more frequently occurs among women and between the ages of 15 and 25 years. It is diagnosed based on the improved diagnostic criteria. Chorea is a disorder characterized by high-amplitude involuntary movements, and although it is the most common symptom of acute rheumatic fever (ARF), it rarely manifests itself as the neuropsychiatric involvement of SLE (1-3).

\section{Case Report}

A 13-year-old girl presented to an emergency pediatric polyclinic due to involuntary movement of her arms and legs that persisted for a week. Choreiform movements were observed; when mild mitral insufficiency was detected on performing echocardiography (ECO), considering Sydenham's chorea due to ARF, penicillin and valproic acid treatment was started after the evaluation of the pediatric neurology. The patient had a normal electroencephalogram and was admitted to our clinic for undergoing further examinations and treatment when an increase in involuntary movements of the face, head, neck, and extremities and complete loss of speech after three days was observed. The patient's and her family's medical history were unremarkable. During her physical examination, her conscious was clear, she understood whatever was said, but she could not speak. She showed mimic-like movements on her face in the form of smelling flowers and moving her lips to the right and left. She also showed common uncontrolled choreiform movements throughout the body, ptosis in the right eye, ataxic walking, and an inability to stand. The pupillary light reflex was $+/+$; her deep tendon reflexes were 
hypoactive and muscle strength was normal, and there were no meningeal irritation findings and pathological reflexes. Her complete blood count revealed the following: leukocyte count: $5000 / \mathrm{mm}^{3}$, hemoglobin level: $10.5 \mathrm{~g} / \mathrm{dL}$, hematocrit: $30.7 \%$, and thrombocyte count: $163,000 / \mathrm{mm}^{3}$. The patient's electrolyte, kidney, and liver test results were normal. As biochemical examinations, her C-reactive protein (CRP) tested negative and erythrocyte sedimentation rate (ESR) was 12 $\mathrm{mm} / \mathrm{h}$. The patient had an antistreptolysin O (ASO) titer of less than $50 \mathrm{IU} / \mathrm{mL}$ and a sterile throat culture. On performing cerebral magnetic resonance imaging, ischemic changes showing hyperintense signal features were detected in $\mathrm{T} 2$ and FLAIR images in the deep watershed area in the left frontoparietal region. In two days, the patient's symptoms became more serious; however, the patient had no rash and aphtha on performing examinations and a history of the same in her medical records, negative acute phase markers in examinations performed, and no features except for anemia on performing examinations. Despite negative acute-phase responses, vasculitis tests were performed; however, because encephalitis could not be excluded, after viral and autoimmune encephalitis tests were performed, intravenous immunoglobulin (IVIG) and high-dose $(30 \mathrm{mg} / \mathrm{kg} /$ day) methylprednisolone therapy were initiated for treating autoimmune encephalitis. Leucopenia, thrombocytopenia, and lymphopenia developed, and the ESR increased to $43 \mathrm{~mm} / \mathrm{h}$ during the examinations performed the following day; proteinuria was detected in her complete urine examination, and aphtha was observed in her mouth. Antinuclear antibody, anti-ds DNA antibody, antineutrophil cytoplasmic antibody, and anti-ENA Sm Antibody tested positive, whereas anticardiolipin IgM-IgG antibody, anti-ribosomal protein, and lupus anticoagulant tested negative. The complement component 3 level was low at 30 $\mathrm{mg} / \mathrm{dL}$, and complement component 4 level was low at 5.6 $\mathrm{mg} / \mathrm{dL}$. In 24-h urine samples, the protein level was $276 \mathrm{mg} /$ day, but the samples were collected after the administration of high-dose methylprednisolone three times. The patient's eye examination result was normal. The kidney biopsy result of the patient who met the SLE criteria was compatible with class 2 SLE nephritis. In the first week, under IVIG, methylprednisolone, cyclophosphamide, and hydroxychloroquine therapy, the patient's movement disorders completely disappeared and her speech returned to normal. Even though the ASO titer was normal, secondary benzathine penicillin prophylaxis and infective endocarditis prophylaxis were recommended by the cardiologist because mitral insufficiency was seen on performing ECO. The treatment and follow-up of the patient has been continuing in the pediatric nephrology polyclinic.

\section{Discussion}

Neuropsychiatric symptoms are seen in $25-95 \%$ of children with SLE, and the most common symptom is headache.
Less frequently, neuropathy, myasthenia gravis, myelopathy, and movement disorders are seen. The most common type of movement disorder is chorea, and the child age group has been shown as $0-5 \%$ in the course of SLE in different series (1-4).

Movement disorders are thought to be related to multifactorial causes in SLE. It has been reported that immune complexes, autoantibodies, and cytokines cause neurological disorders in SLE with direct effects and that vasculopathy developing in arterioles that feed the basal ganglia, coagulopathy, and bleeding disorders cause neurological disorders with indirect effects $(2,5)$.

Chorea is characterized by sudden involuntary movements that can be seen in any part of the body. It may occur in the extremities, face, neck, and trunk. Sydenham's chorea, which is one of the major cause of ARF, is the most common cause of chorea in children $(2,6)$. Other causes include cerebrovascular diseases, hyperthyroidism, poisoning, Huntington's disease, and collagen vascular diseases such as SLE (7). Chorea may occur at any time of SLE; it may also be a symptom for presenting to a clinic $(2,3)$. Our patient presented due to involuntary movements that started at the extremities. Because our patient presented due to only chorea; because the CRP level, ESR, and WBC count were normal at presentation; and because mild mitral insufficiency was detected on performing ECO, treatment was initiated considering Sydenham's chorea in our patient. Within days, there was a considerable increase in the number of complaints, and the severity of involuntary movements spreading across the face, neck, and body increased; the patient was unable to speak and walk. The number of acute-phase responses increased, and aphtha in the mouth and positivity in vasculitis test results associated with SLE occurred.

Many studies have described a close relationship between chorea that is seen in patients with SLE and antiphospholipid antibodies $(1,2,7,8)$. In our case, antiphospholipid antibodies tested negative.

\section{Conclusion}

Chorea is a well-known, but rarely encountered, clinical picture that appears as a symptom of SLE for presenting to a clinic. In the differential diagnosis of patients with isolated chorea, neuropsychiatric-onset SLE should be kept in mind in addition to ARF, and even though acute-phase responses and the hematological picture are not compatible in the beginning, evaluations should be repeated if necessary.

Informed Consent: Informed consent was obtained from patients who participated in this study.

Peer-review: Externally peer-reviewed. 
Author Contributions: Concept - A.D.D., N.G.; Supervision - A.D.D.; Data Collection and/or Processing - A.D.D., T.U.Ş., U.E.; Literature Review - M.K., N.G.; Writing - A.D.D., M.K.; Critical Review - N.G., T.U.Ş.

Conflict of Interest: No conflict of interest was declared by the authors.

Financial Disclosure: The authors declared that this study has received no financial support.

\section{References}

1. Mirabelli G, Cannarile F, Bruni C, Vagelli R, De Luca R, Carli L. One year in review 2015: systemic lupus erythematosus. Clin Exp Rheumatol 2015; 33: 414-25.

2. Torreggiani S, Torcoletti M, Cuoco F, Di Landro G, Petaccia A, Corona F. Chorea, a little-known manifestation in systemic lupus erythematosus: short literature review and four case reports. Pediatr Rheumatol Online J 2013; 11: 36. [CrossRef]

3. Sanna G, Bertolaccini ML, Cuadrado MJ, Laing H, Khamashta MA, MathieuA, et al. Neuropsychiatric manifestations in systemic lupus ery- thematosus: prevalence and association with antiphospholipid antibodies. J Rheumatol 2003; 30: 985-92.

4. Joseph FG, Lammie GA, Scolding NJ. CNS lupus: a study of 41 patients. Neurology 2007; 69: 644-54. [CrossRef]

5. Moore PM, Lisak RP. Systemic lupus erythematosus: immunopathogenesis of neurologic dysfunction. Springer Semin Immunopathol 1995; 17: 43-60. [CrossRef]

6. Swedo SE, Leonard HL, Schapiro MB, Casey BJ, Mannheim GB, Lenane MC, et al. Sydenham's chorea: physical and psychological symptoms of St Vitus dance. Pediatrics 1993; 91: 706-13.

7. Poil AR, Yousef Khan F, Lutf A, Hammoudeh M. Chorea as the first and only manifestation of systemic lupus erythematosus. Case Rep Rheumatol 2012; 907402 [CrossRef]

8. Arisaka O, Obinata K, Sasaki H, Arisaka M, Kaneko K. Chorea as an initial manifestation of systemic lupus erythematosus. A case report of a 10-year-old girl. Clin Pediatr (Phila) 1984; 23: 298-300. [CrossRef] 